\title{
Model for hands-on tonsillectomy surgical training of postgraduate residents during COVID-19 pandemic
}

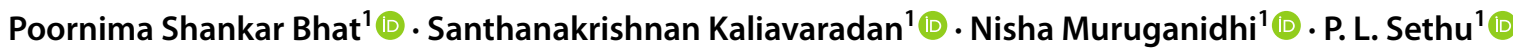

Received: 8 August 2020 / Accepted: 15 September 2020 / Published online: 26 September 2020

(c) Springer-Verlag GmbH Germany, part of Springer Nature 2020

\begin{abstract}
Purpose Otorhinolaryngological surgeries being highly aerosol-generating carry the risk of transmission of SARS-CoV-2. Most centers have canceled elective surgeries. Surgical demonstration and hands-on training of postgraduate residents are challenging during the COVID-19 pandemic. Continued training of residents is of utmost importance in terms of time and skill development. Tonsillectomy is one of the most common and important surgeries in ENT. Resident training of tonsillectomy is essential. Here, we present a simple, inexpensive model for tonsillectomy hands-on training addressing critical steps of the surgery.

Methods An oropharynx and tonsil model is prepared using easily available materials, such as a small plastic tub, gauze pieces, ribbon gauze, and rubber strips. The junior residents are trained in all the critical steps of tonsillectomy using routine tonsillectomy instruments.

Conclusion This model for tonsillectomy is a simple, inexpensive model for training postgraduate residents during and after the COVID-19 pandemic. We hope that this model can help young residents to get trained in critical steps of tonsillectomy, in a closely simulated environment and help them develop confidence and skills in managing live patients.
\end{abstract}

Keywords Model $\cdot$ Tonsillectomy $\cdot$ COVID-19 $\cdot$ Surgical training $\cdot$ Postgraduate

\section{Introduction}

Postgraduation in surgical courses largely depends on observation and training which contributes to skill development. COVID-19 pandemic has adversely affected medical education all over the world, especially surgical specialties. Theory and some aspects of the practical teachings are being conducted online through webinars, online classes, etc., whereas surgical demonstrations are mostly relying on prerecorded videos. Live surgical demonstrations and handson training which are crucial for surgical skills are lagging. Tonsillectomy is one of the major and commonly performed surgeries in Otorhinolaryngology. Demonstration and training of junior residents in the postgraduation course are of utmost importance.

Santhanakrishnan Kaliavaradan

santhanakrishnan2709@gmail.com

1 Department of Otorhinolaryngolgy, Sri Manakula Vinayagar Medical College and Hospital, Kalitheerthalkuppam,

Madagadipet, Puducherry 605107, India
In otorhinolaryngology, clinical examination and invasive procedures on the respiratory tract and connected cavities expose people to direct transmission of SARS-CoV-2 by inhalation or ocular projection of contaminated droplets, and to indirect transmission by contact with contaminated hands, objects, or surfaces [1].

Most centers have canceled non-cancer elective procedures following international recommendations [2]. This adversely affects the training of surgical residents which can impair their clinical confidence and performance.

Among different and latest techniques of tonsillectomy, cold steel tonsillectomy using ties and /or packs is the technique with by far the lowest risk of postoperative hemorrhage (1.3\%) and return to theater (1.0\%) [3]. To demonstrate and to provide hands-on training to postgraduate students in this time tested technique, a simple and cost-effective model is developed which can help in understanding the concepts and developing skills in this surgery. 


\section{Materials and methods}

A model for tonsillectomy training was prepared using readily available materials, to train the postgraduate students in the cold steel tonsillectomy, by dissection and snare method.

Materials required for preparing the model:-

- Plastic tub about $10 \times 12 \mathrm{~cm}$ size.

- Red color ink.

- $6 \times 6 \mathrm{~cm}-$ long ribbon gauze-2, colored in red ink and dried.

- Gauze pieces-4, colored in red ink and dried.

- Adhesive plaster.

- Adhesive tape.

- Cotton/silk thread.

- Blade.

- Old endotracheal tube.
A plastic tub is taken and the base is removed using a blade. Gauze pieces are rolled into an oval shape and tied using ribbon gauze, representing tonsil about $3 \times 2 \times 2$ cm size (Fig. 1a). A thin strip of rubber (eraser) around $2 \times 4 \times 15 \mathrm{~mm}$ size is cut and pasted over the backside of the pseudotonsil, such that about $6 \mathrm{~mm}$ of the rubber strip is projecting outwards (Fig. 1b). Two such pseudotonsils are prepared. In the lower end of the tubs on either side, a double-layer gauze piece is pasted to represent the tonsillar fossa.

To simulate the mucosa of the oral cavity and oropharynx, the inner surface of the tub is plastered with pale pinkcolored elastic adhesive bandage.

The rubber strip of the pseudotonsil is pasted to the lower pole of tonsillar fossa using adhesive gum, representing the lower pole pedicle. Ribbon gauze is pasted in oval shape over the tonsil bed, representing anterior and posterior pillars. Pseudotonsil is snugly fit into the tonsillar fossa, using a double-side adhesive tape. The model is now ready to use (Fig. 2a and b).
Fig. 1 a and $\mathbf{b}$ Preparing the pseudotonsil using gauze piece and attaching the rubber strip. a Preparing the pseudotonsil using gauze piece. b Attaching the rubber strip over the backside of pseudotonsil
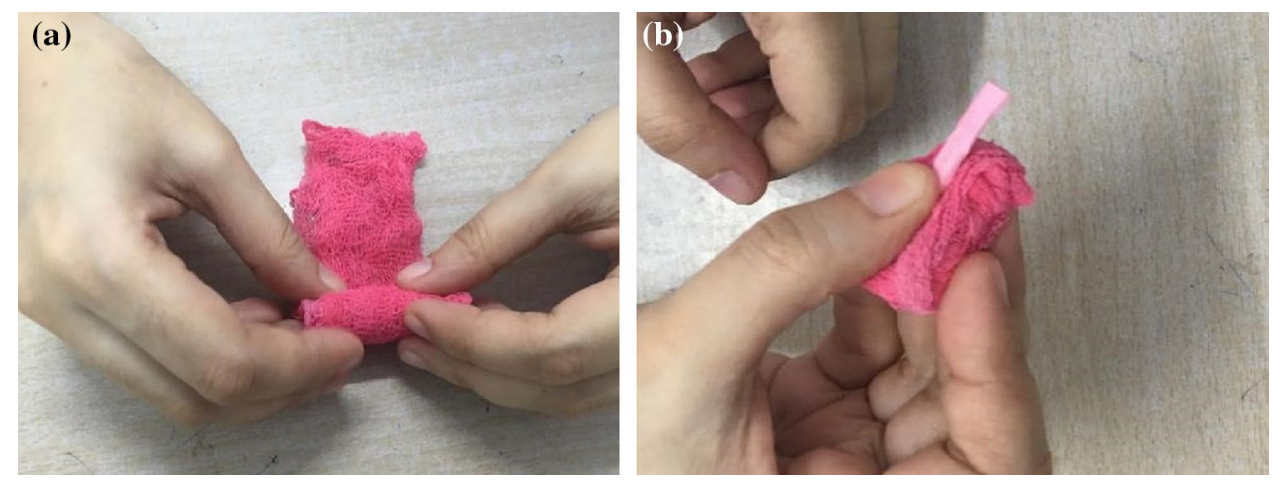
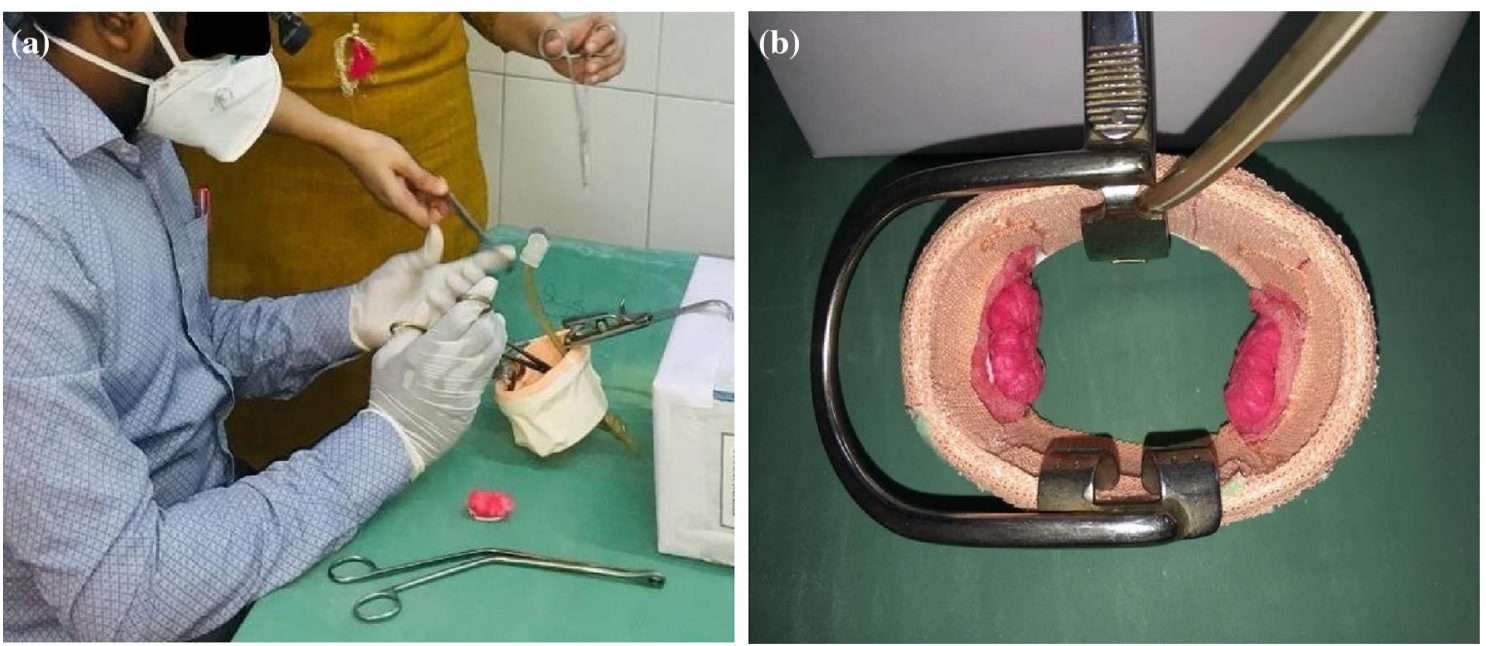

Fig. 2 a and $\mathbf{b}$ Tonsil model and position ready to use. a Position of the model and resident. $\mathbf{b}$ Model ready to use 
Three ENT surgeons in the department well experienced in tonsillectomy surgeries were invited to use the tonsillectomy model and to provide feedback regarding improvisations in the model in comparison to live surgery. According to their feedback, modifications in the design of the model were made. The final design of the model was used in the training of postgraduate residents.

The resident is now trained to mount the model on a Boyle-Davis mouth gag, over an old endotracheal tube and stabilize it on a box. Then, the resident grasps the tonsil using Denis-Brown tonsil holding forceps and starts to dissect the tonsil from the upper pole, till the lower pole using Mollison's tonsil dissector. The tactile sensation obtained during dissection between adhesive tape and the tonsillar fossa gauze closely simulates that of tonsil dissection in the peritonsillar space. Dissection stops at the lower pole (Fig. 3).

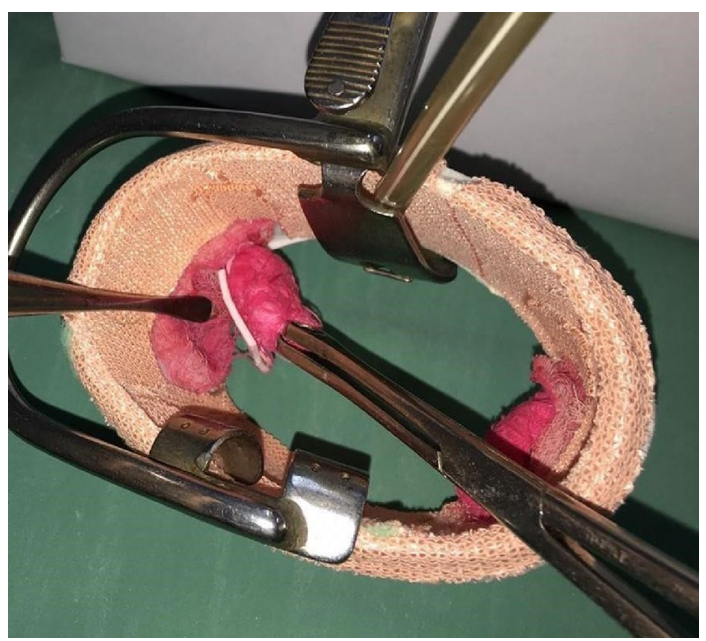

Fig. 3 Dissection of tonsil in peritonsillar space
Then, the resident practices applying Eve's tonsillar snare to the lower pole of the tonsil represented by the rubber (eraser) strip, and the crushing and cutting of lower pole is performed (Fig. 4a and b).

The cutting of the thin rubber strip closely resembles the sensation of using Eve's snare on the lower pole of tonsil in live patients. Other methods of tonsillectomy like clipping and tying of lower pole can also be practiced at this stage.

Another most critical step is applying ligature to the bleeding vessels. The lower pole of the tonsil is held using Birkett's first artery forceps and Negus second artery forceps are applied. Ligation is done using silk threads (Fig. 5a and b). Multiple numbers of ligations are performed over tonsillar fossa. The same procedure is practiced on both sides.

Achieving the removal of tonsil using Eve's snare and proper application of ligatures acts as a self-assessment tool for the resident, and this procedure can be repeated any number of times in the same model.

Six postgraduate residents used the model for training in tonsillectomy and gave their feedback on a five-point scale from 1 to 5, 5-excellent, 4-good, 3-fair, 2-poor, and 1 -bad (Table 1). 15 items on the important steps of tonsillectomy, understanding the concepts, overall satisfaction, etc. were included. For a maximum score of 75 , an average of $67.83(90.4 \%)$ was obtained. Understanding the concepts was excellent for all the trainees. Overall satisfaction of the model was excellent for five of the trainees and good for one trainee. Training with ET tube in situ, dissection in the peritonsillar space, Eve's snare application, crushing and cutting of lower pole, and overall satisfaction of using the model received maximum points on feedback. Simulation of bleeding during the procedure was fair uniformly and further modifications to include active bleeding simulator can enhance the experience.
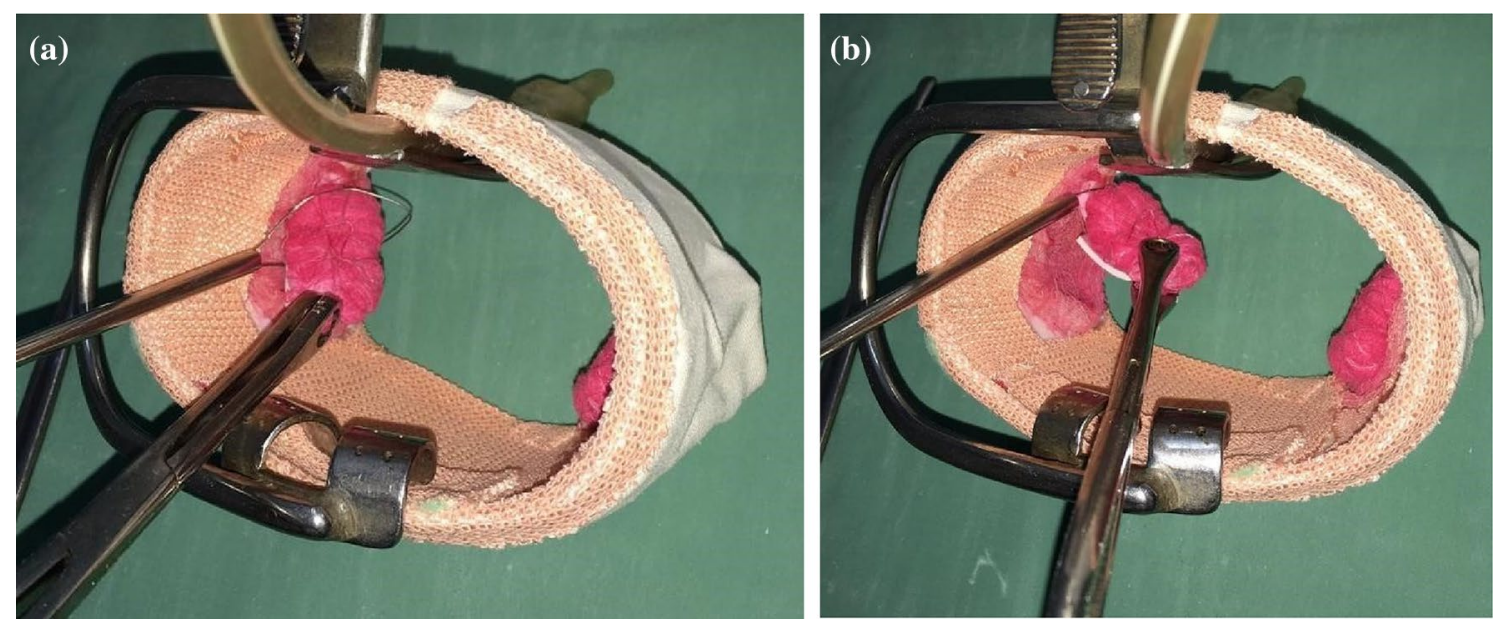

Fig. 4 a and $\mathbf{b}$ Application of Eve's tonsillar snare. a Application of Eve's tonsillar snare. b Crushing and cutting of lower pole using the snare 

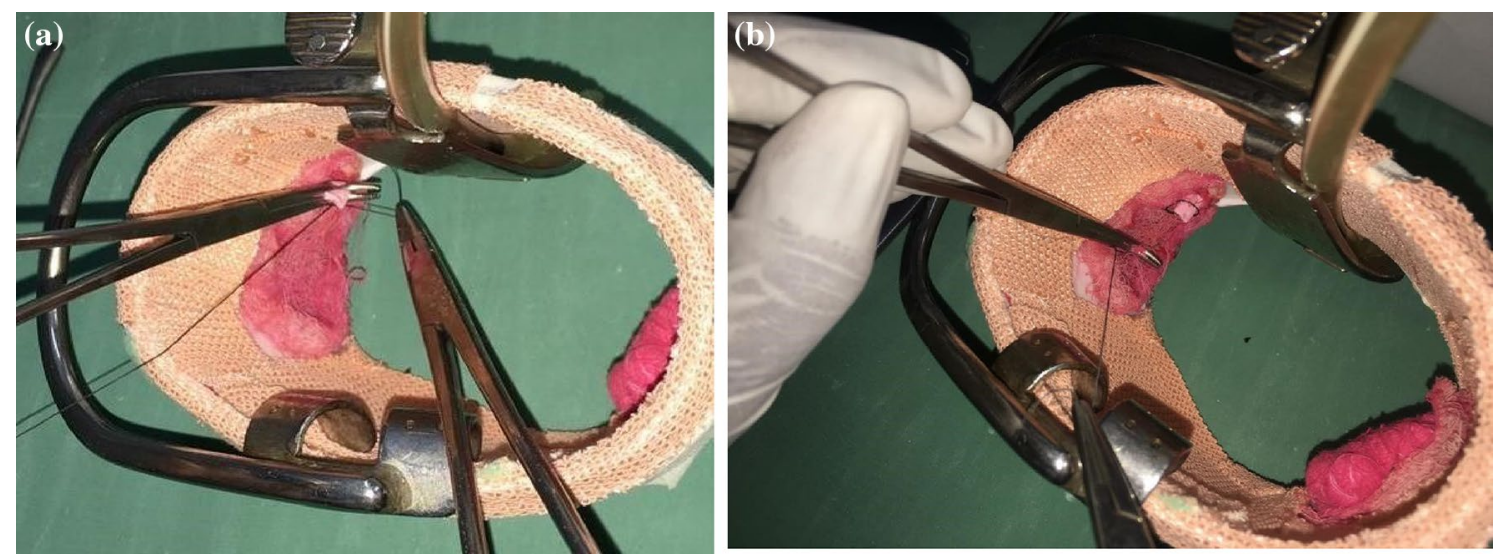

Fig. 5 a and $\mathbf{b}$ Ligation of lower pole and tonsillar fossa bleeders. a Ligation of the lower pole. b Ligation of bleeding vessels in the tonsillar fossa

Table 1 Summary of feedbacks of six postgraduate residents on a five-point scale based on their experience with tonsillectomy model for training

\begin{tabular}{|c|c|c|c|c|c|c|c|}
\hline Steps performed using the model & PG1 & PG2 & PG3 & PG4 & PG5 & PG6 & Average score $(\%)$ \\
\hline 1. Simulation of oropharynx & 4 & 4 & 5 & 4 & 5 & 4 & $4.33(86.7 \%)$ \\
\hline 2. Simulation of Rose's position & 4 & 5 & 5 & 4 & 4 & 5 & $4.5(90 \%)$ \\
\hline 3. Application of Mouth gag & 5 & 4 & 5 & 4 & 5 & 4 & $4.5(90 \%)$ \\
\hline 4. Training with ET tube in situ & 5 & 4 & 5 & 5 & 5 & 5 & $4.83(96.67 \%)$ \\
\hline 5. Simulation of tonsils & 4 & 4 & 5 & 4 & 4 & 4 & $4.16(83.33 \%)$ \\
\hline 6. Usage of tonsillectomy instruments & 5 & 5 & 5 & 5 & 4 & 4 & $4.67(93.3 \%)$ \\
\hline $\begin{array}{l}\text { 7. Finding the plane of dissection near } \\
\text { upper pole }\end{array}$ & 5 & 5 & 4 & 5 & 5 & 4 & $4.66(93.3 \%)$ \\
\hline 8. Dissection in peritonsillar space & 5 & 5 & 4 & 5 & 5 & 5 & $4.83(96.67 \%)$ \\
\hline 9. Eve's snare application & 5 & 5 & 5 & 5 & 5 & 4 & $4.83(96.67 \%)$ \\
\hline 10. Cutting and crushing of lower pole & 5 & 5 & 5 & 5 & 4 & 5 & $4.83(96.67 \%)$ \\
\hline 11. Ligation of lower pole & 5 & 5 & 4 & 4 & 4 & 5 & $4.5(90 \%)$ \\
\hline $\begin{array}{l}\text { 12. Simulation of bleeding during pro- } \\
\text { cedure }\end{array}$ & 3 & 3 & 3 & 3 & 3 & 3 & $3(60 \%)$ \\
\hline $\begin{array}{l}\text { 13. Ligation of bleeding vessels in tonsil- } \\
\text { lar fossa }\end{array}$ & 4 & 4 & 5 & 4 & 5 & 4 & $4.33(86.67 \%)$ \\
\hline $\begin{array}{l}\text { 14. Understanding the concepts of tonsil- } \\
\text { lectomy }\end{array}$ & 5 & 5 & 5 & 5 & 5 & 5 & $5(100 \%)$ \\
\hline $\begin{array}{l}\text { 15. Overall satisfaction with tonsillec- } \\
\text { tomy training }\end{array}$ & 5 & 5 & 5 & 5 & 5 & 4 & $4.83(96.67 \%)$ \\
\hline Total score $(\%)$ & $69(92 \%)$ & $68(90.67 \%)$ & $70(93.33 \%)$ & $67(89.33 \%)$ & $68(90.67 \%)$ & $65(86.67 \%)$ & $67.83(90.4 \%)$ \\
\hline
\end{tabular}

5-excellent, 4-good, 3-fair, 2-poor, and 1-bad

$P G$ postgraduate resident

\section{Discussion}

Surgical skill development depends both on observation and hands-on practice in a simulated environment, before performing on live patients. Visual familiarity with anatomy and hand dexterity are most important in tonsillectomy. This model can help the residents to practice the procedure of tonsillectomy in a closely simulated environment.

Important factors addressed in this model are:

1. Visual simulation of oropharynx and tonsils.

2. Application of mouth gag, over ET tube.

3. Position of model mimicking Rose's position. 
4. Practicing usage of all routine tonsillectomy instruments.

5. Finding the right plane near the superior pole of the tonsil.

6. Dissection of tonsils from the tonsillar fossa.

7. Applying Eve's tonsillar snare and crushing and cutting the lower pole of the tonsil.

8. Ligation of bleeding vessels.

Some different models have been used in the past to train residents in tonsillectomy. However, most of the models were very simple in design and focussed mainly on the practice of ligation of lower pole or vessels in the tonsillar fossa.

To train the residents in tonsillar ligations, a model was developed using a tissue paper box. It was a very simple and inexpensive model, focussing on tonsil knot tying only [4]. They used an empty cardboard tissue paper box, at the bottom of which two paper clips and hooked wires were fixed. Tonsil tying was practiced on the hooked wires. Simulation of oropharynx and tonsils, important steps like dissection in peritonsillar space, and removal of tonsils were not addressed. Our model closely mimics the oropharynx and tonsils in terms of appearance, and the trainee can practice almost all the crucial steps of tonsillectomy as listed above.

In another model using a foam cup, training in tonsil vessel ligation was done using red color-stained gauze pieces at the bottom of the cup [5]. This model also focussed on training of ligation only and did not address any other important steps of tonsillectomy.

In a model of tonsil tie simulator, a commercially available airway demonstration model was used and fingers of disposable latex gloves were used to represent tonsil [6]. This model included features like the simulation of the oropharynx and using red food dye as blood, but the pseudomouth model had to be bought commercially and this focuses mainly on ligation of the lower pole only. Our model is inexpensive and can be prepared using readily available materials and trains the residents in maximum steps of tonsillectomy.

Another commercially available model, AirSim Bronchi, was also used to train the junior residents, to increase realism, familiarization with more number of equipment [7]. This model requires a high initial investment. Replica tonsils were prepared using gauze and focussed mainly on the lower pole ligation. Other steps of tonsillectomy were not addressed.

A simple model designed recently in South India, using a plastic cup, sponge, and cotton balls [8]. In this model, a plastic cup was used as the oropharynx, red ink-stained cotton balls were used as tonsils, and some more steps were incorporated like the dissection of tonsils (cotton balls) from the superior constrictor (plastic sheet over sponge) and ligation of vessels. Important steps like the application of mouth gag and cutting of the lower pole of tonsil using snare were not addressed.

Our model is different in closely simulating all the crucial surgical steps of tonsillectomy and being inexpensive and easy to set up. The main highlight being the tactile sensation obtained during peritonsillar space dissection and while cutting the lower pole of the tonsil using snare which closely mimics that in live surgery.

This model can help in continuing surgical training of residents in tonsillectomy during and after the COVID-19 pandemic. The same model can also be used in undergraduate teaching, to explain the steps and concepts of tonsillectomy. Prospective studies, using this model for training a large number of postgraduate students, and comparison with their performance during live surgeries under guidance in the future can help in further improvisations in the model and it can become an important tool in surgical training of young residents.

\section{Conclusion}

This model for tonsillectomy training is a simple, inexpensive model for training postgraduate residents during and after the COVID-19 pandemic. We hope that this model can help young residents to get trained in critical steps of tonsillectomy, in a closely simulated environment and help them to develop confidence and skills in managing live patients.

Acknowledgements We acknowledge our postgraduate students and peers for their valuable feedback on the model.

Funding No funding received.

Data availability Not applicable.

Code availability Not applicable.

\section{Compliance with ethical standards}

Conflicts of interest The authors declare that they have no conflicts of interest.

Ethical approval The model did not involve any human or animal participants. It was in accordance with the ethical standards of the institutional research committee of Sri Manakula Vinayagar Medical College and Hospital, Puducherry, India.

Informed consent Informed consent was obtained from all the postgraduate students who participated in the training.

\section{References}

1. Couloigner V, Schmerber S, Nicollas R, Coste A, Barry B, Makeieff M et al (2020) COVID-19 and ENT surgery. Eur Ann 
Otorhinolaryngol Head Neck Dis 137(3):161-166. https://doi. org/10.1016/j.anorl.2020.04.012

2. Elizabeth Brindle M, Gawande A (2020) Managing COVID-19 in surgical systems. Ann Surg 272(1):e1-e2. https://doi.org/10.1097/ SLA.0000000000003923

3. Royal College of Surgeons of England (2005) National Prospective Tonsillectomy Audit. London: RCS. National Prospective Tonsillectomy Audit: final report. https://www.tonsil-audit.org/ documents/ta_finalreport.pdf. Accessed 31 July 2020

4. Wasson J, De Zoysa N, Stephens J (2009) Tissue-box tonsillar tie trainer. Clin Otolaryngol 34(2):175-176

5. Al-Hussaini A, Addams-Williams J, Tomkinson A (2011) The Cardiff cup: a model for tonsillectomy tie training. Otolaryngology. https://doi.org/10.4172/2161-119X.1000102
6. Duodu J, Lesser T (2013) Tonsil tie simulator. J Laryngol Otol 127(9):924-926. https://doi.org/10.1017/S0022215113001849

7. Douglas J, Senior A, Scott A (2014) The Telford tonsillectomy tie trainer: an AirSim model modification. Ann R Coll Surg Engl 96(8):634-634. https://doi.org/10.1308/rcsann.2014.96.8.634

8. Shankar G, Jugmalram D (2014) A Model for Tonsillectomy. IOSR J Dent Med Sci 13(7):29-33. https://doi.org/10.9790/085313722933

Publisher's Note Springer Nature remains neutral with regard to jurisdictional claims in published maps and institutional affiliations. 